\title{
A microscope for two-dimensional measurements of in vivo chlorophyll fluorescence kinetics using pulsed measuring radiation, continuous actinic radiation, and saturating flashes
}

\author{
H. KÜPPER ${ }^{* * *, * * *}$, I. ŠETLÍK ${ }^{* *, * * *}$, M. TRTÍLEK ${ }^{+}$, and L. NEDBAL ${ }^{++}$ \\ University of Konstanz, Faculty of Sciences, Department of Biology, D-78457 Konstanz, Germamy* \\ Microbiological Institute, Academy of Sciences of the Czech Republic, Department of Autotrophic Microorganisms, \\ Opatovický mlýn, CZ-37981 Trebon̆, Czech Republic ${ }^{* *}$ \\ Biological Faculty, University of South Bohemia, Branišovská 31, CZ-370 05, Ćeské Budẽjovice, Czech Republic ${ }^{* * *}$ \\ Photon Systems Instruments Ltd., Koláckova 31, CZ-62100 Brno, Czech Republic ${ }^{+}$ \\ Institute of Landscape Ecology, Academy of Sciences of the Czech Republic, Photosynthesis Research Centre, Zámek, \\ CZ-37333 Nové Hrady, Czech Republic ${ }^{+}$
}

\begin{abstract}
Transients of chlorophyll fluorescence in photosynthetic objects are often measured using short pulses of exciting radiation, which has recently been employed to capture kinetic images of fluorescence at the macroscopic level. Here we describe an instrument introducing this principle to recording of two dimensional fluorescence transients in microscopic objects. A modified fluorescence microscope is equipped with a CCD camera intensified by a micro-channel plate image amplifier. The microscopic field is irradiated simultaneously by three types of radiation: actinic radiation, saturating flashes, and pulsed measuring radiation. The measuring pulses are generated by a light-emitting diode and their duration is between 10 to $250 \mu \mathrm{s}$. The detection of fluorescence images $(300 \times 400$ pixels, 8 bit) has a maximum time resolution of $40 \mathrm{~ms}$ and is gated in synchrony with the exciting pulses. This allows measuring on a background of a continuous actinic radiation up to irradiance that can elicit the maximal fluorescence yield $\left(F_{M}\right)$. On the other hand, the integral irradiance of the objects by the measuring radiation is very low, e.g, $0.08 \mu \mathrm{mol} \mathrm{m} \mathrm{m}^{-2} \mathrm{~s}^{-1}$ at $0.5 \mu \mathrm{m}$ spatial resolution and $0.006 \mu \mathrm{mol}$ $\mathrm{m}^{-2} \mathrm{~s}^{-1}$ at $4 \mu \mathrm{m}$ spatial resolution. This allows a reliable recording of $\mathrm{F}_{0}$ even in very short time intervals $(e . g ., 5 \times 80 \mathrm{~ms})$. The software yields fluorescence kinetic curves for objects in user-selected areas as well as complete false-colour maps of the essential fluorescence kinetics parameters $\left(F_{M}, F_{0}, F_{V}, F_{V} / F_{M}\right.$, etc.) showing a two-dimensional distribution of their values. Several examples demonstrate that records of fluorescence kinetics can be obtained with a reasonable signal-tonoise ratio with all standard microscope objectives and with object sizes reaching from segments of leaf tissue to individual algal cells or chloroplasts.
\end{abstract}

\footnotetext{
Received 6 December 2000, accepted 27 February 2001.

"*Author for correspondence; fax: ++420-333-721246; e-mail: Setlik@alga.cz

Abbreviations: $\mathrm{CCD}=$ charge-coupled device, the light detector of digital video cameras; $\mathrm{Chl}=$ chlorophyll; $\mathrm{FKM}=$ fluorescence kinetic microscope, i.e., a microscope allowing two-dimensional (= imaging) records of fluorescence kinetics; $F_{0}=$ minimal fluorescence yield of a dark adapted sample, fluorescence in non-actinic measuring radiation; $F_{0}{ }^{\prime}=$ minimal fluorescence yield of a light-adapted sample, fluorescence in non-actinic radiation after actinic irradiation; $F_{M}=$ maximum fluorescence yield of a dark adapted sample; $\mathrm{F}_{\mathrm{M}}{ }^{\prime}=$ maximum fluorescence yield of a light-adapted sample, during the exposure to actinic irradiation, and reduced by non-photochemical quenching; $F_{p}=$ fluorescence yield at the peak level of the induction curve after the onset of actinic irradiation; $\mathrm{F}_{\mathrm{s}}=$ steady state fluorescence under the given actinic irradiation, i.e., after the end of the induction transient; $F_{\mathrm{V}}=$ variable fluorescence $F_{Y}=F_{M}-F_{0}$; LED = light-emitting diode; $M C P=$ micro-channel plate, the device in generation 2 and 3 image intensifiers which amplifies the signal; PS2 = photosystem 2; $q_{P}=$ photochemical quenching $=\left(F_{M}^{\prime}-F\right) /\left(F_{M}^{\prime}-F_{0}^{\prime}\right) ; q_{N P}=$ nonphotochemical quenching $=1-q_{p} ; \Phi_{F}=$ fluorescence yield: mol(emitted fluorescence photons)/mol(absorbed excitation photons); $\Phi_{\mathrm{PS} 2}=\mathrm{PS} 2$ efficiency measured as $\mathrm{F}_{\mathrm{V}} / \mathrm{F}_{\mathrm{M}}$.

Acknowledgements: We are obliged to T. Macho for carrying out all precision mechanical works during the construction of the FKM and the measuring chamber. We thank M. Hlasek for construction of some electronic parts that made the operation of the system easier, for effective help with the measurements to E. Setlikova, with growing the cultures to $K$. Bísová, with evaluation of the data to L. Oborský, and with providing laboratory equipment to D. Stys. We thank F.C. Kupper and C. Gachon for good suggestions during the preparation of the manuscript. The providing of the fluorescence microscope by V. Zachleder, the Ectocarpus culture by D.G. Müller and I. Maier, and the strain of Micrasterias by A. Couté is most gratefully acknowledged. The work was supported by a grant VS96085 from the Department of Education of the Czech Republic and by the Studienstiftung des deutschen Volkes.
} 
Additional key words: Ectocarpus; Elodea; fluorescence imaging; fluorescence quenching analysis; Hibiscus; photosynthetic heterogeneity; photosystem 2; Scenedesmus; topography of fluorescence induction.

\section{Introduction}

The method of chlorophyll (Chl) fluorescence measurement is widely applied in most diverse fields of photosynthesis research, from biophysical investigations into the mechanism of primary reactions to ecophysiological studies on leaves and plant stands (reviewed by Krause and Weis 1991, Dau 1994, Govindjee 1995). This is mainly because the fluorescence signal can be detected and analysed over many orders of magnitude as to the time scale (ps to h) and as to the object size (photosystem particles to plant stands). The universality of the method helps to inter-relate events at these vastly different levels. The potential of the method has been further stimulated in the last decade by introduction of the imaging detectors (CCD cameras and MCP image intensifiers) which provide a time sequence of two-dimensional maps of the fluorescence emission. This allows simultaneous evaluation of kinetic response in various segments of macroscopic or microscopic objects that reveal, in two dimensions, the frequent spatial heterogeneity (Omasa et al. 1987, Meyer and Genty 1988, Fenton and Crofts 1990, Cardon et al. 1994, Rolfe and Scholes 1995, Osmond et al. 1999, Lootens and Vandecasteele 2000).

Chl fluorescence kinetic imaging is applied mainly to analyse physiological questions. Consequently the focus is on parameters indicating the physiological state of the photosynthetic organism investigated. Among these, the key positions are occupied by $(I)$ the maximum value of the fluorescence yield attained under saturating irradiance $\left(F_{M}\right)$, when the plastoquinone pool between the photosystems is fully reduced, and (2) the minimum value of fluorescence yield detected under extremely weak irradiance $\left(\mathrm{F}_{0}\right)$ which does not noticeably alter the oxidation-reduction poise in the photosynthetic electron transport chain. In this state the plastoquinone pool between the photosystems is largely oxidised.

The assessment of fluorescence under such widely different irradiances is best performed by measuring fluorescence yield in response to short (tens of $\mu \mathrm{s}$ ) measuring radiation pulses of standard strength applied at an appropriate frequency. The application of this

\section{Materials and methods}

Although the system has been primarily developed for the measurement of in vivo $\mathrm{Chl} a$ fluorescence transients, it can also be used to measure kinetics of any other fluorescence with excitation and emission wavelengths principle (Bilger and Schreiber 1990, Schreiber et al. 1993, 1995) promoted in the last decades of the last century fluorescence measurements to the universal method of investigating the photosynthetic apparatus (Govindjee 1995). Unfortunately, it is hard to use this concept in the imaging technique because the detection sensitivity and signal to noise ratio of the cameras are barely sufficient for detecting the response to weak microsecond radiation pulses. Therefore it has often been considered unrealistic to record images of $F_{0}$ in this way (Bowyer et al. 1998). Until now, $\mathrm{F}_{0}$ images were mostly captured by using a continuous low-irradiance treatment given over a long image-integration period (Meyer and Genty 1988, Fenton, and Crofts 1990). Alternatively, a more rapid measurement with a non-imaging modulatedradiation instrument was done in parallel to imaging to extrapolate true $F_{0}$ levels (Daley et al. 1989, Daley 1995, Siebke and Weis 1995, Jensen and Siebke 1997). A kinetic extrapolation to zero-time using a clever formalism has been applied by Oxborough and Baker (1997a, b). All these approaches can, in many situations, yield reliable values of fluorescence parameters required for estimation of the photochemical activity of photo-system 2 (PS2), etc. But they cannot reveal the full and true course of the transients (e.g., light-dark and dark-light).

An application of pulse-modulated excitation for imaging remained an attractive alternative and has been attempted as soon as sufficiently rapid gating and highsensitivity detection systems were available. Lichtenthaler et al. (1996) and Buschmann and Lichtenthaler (1998) tested this approach using a pulsed laser beam expanded to hit a leaf surface. They mapped the $\mathrm{Chl}$ fluorescence yield and the green and blue fluorescence yield of ferulic acids in plants under daylight. Recently, Nedbal et al. (2001) used the pulse-modulated excitation from light emitting diodes (LEDs) for kinetic imaging of $\mathrm{Chl}$ fluorescence transients including $\mathrm{F}_{0}$ records, and also for the detection of the maximal yields $F_{M}$ and $F_{M}$ ' in plants exposed to a broad daylight.

In the present paper we describe the extension of this measuring principle to the microscopic level. within the range compatible with its construction. For this reason, the invariable parts of the system are described first, and specifications related to Chl fluorescence are given in the section "Measuring conditions". 

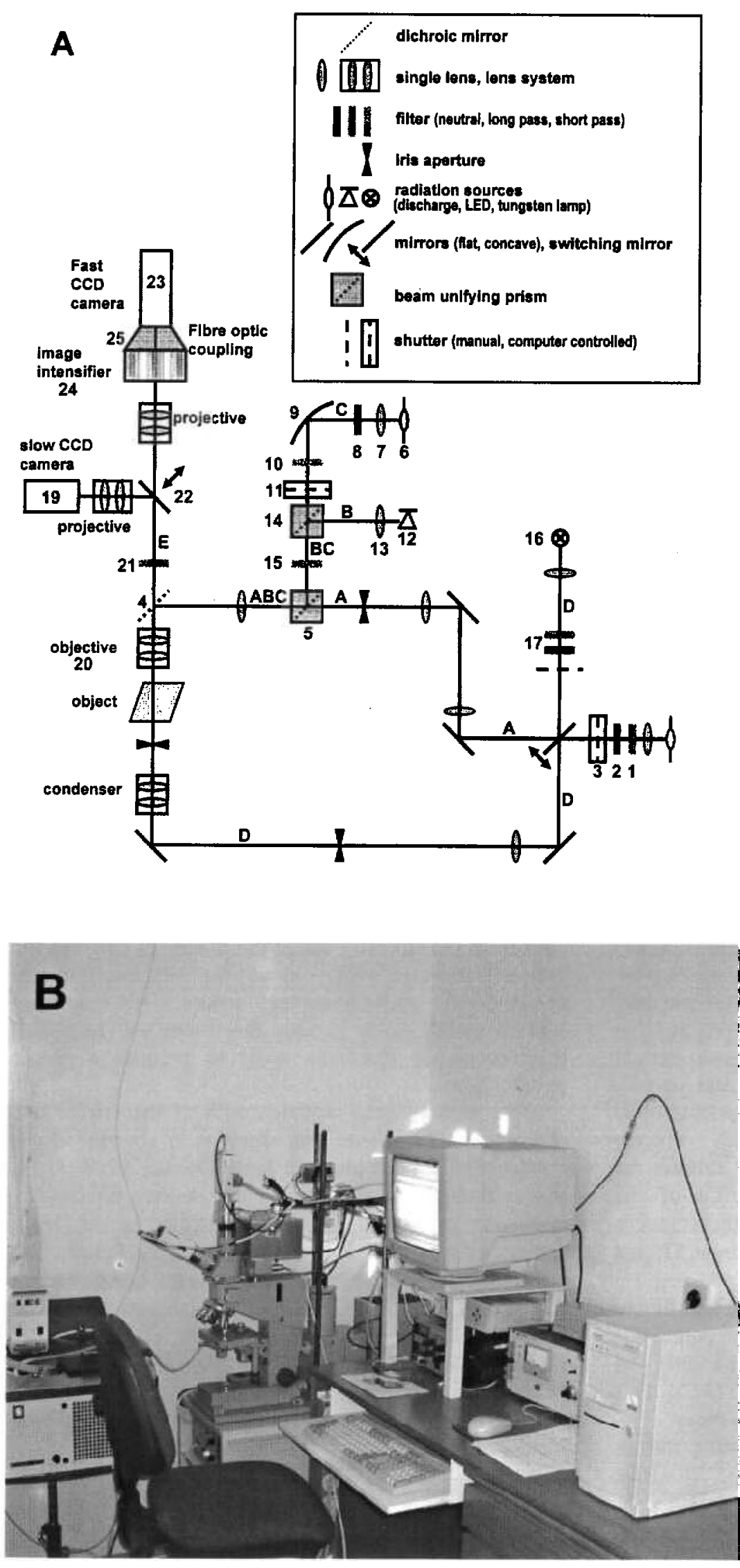

Fig. 1. The fluorescence kinetic microscope. $A$ : schematic representation of the light paths. All numbers and letters are explained in the text. $B$ : photograph of the system. 
Table 1. Maximum irradiances on the sample level achievable with the applied set of filters. "With $100 \mu \mathrm{s}$ flashes in $320 \mathrm{~ms}$ intervals, i.e., the maximum irradiance applied for $F_{0}$ measurements. With the $25-100 \times$ lenses, usually shorter flash times (10-30 $\left.\mu \mathrm{s}\right)$ and correspondingly lower irradiances were used.

\begin{tabular}{lllcc}
\hline Lens & $\begin{array}{l}\text { Light field diameter } \\
{[\mathrm{mm}]}\end{array}$ & $\begin{array}{l}\text { Measuring irradiance } \\
{\left[\mu \mathrm{mol} \mathrm{m} \mathrm{s}^{-1}\right]}\end{array}$ & $\begin{array}{l}\text { Actinic irradiance } \\
{\left[\mu \mathrm{mol} \mathrm{m}^{-2} \mathrm{~s}^{-1}\right]}\end{array}$ & $\begin{array}{l}\text { Saturating irradiance } \\
{\left[\mu \mathrm{mol} \mathrm{m} \mathrm{m}^{-2} \mathrm{~s}^{-1}\right]}\end{array}$ \\
\hline $6.3 \times / 0.20$ & 2.90 & 0.006 & 686 & 524 \\
$16 \times / 0.40$ & 1.06 & 0.026 & 2835 & 2167 \\
$25 \times / 0.63$ & 0.67 & 0.075 & 8295 & 6332 \\
$40 \times / 0.95$ & 0.38 & 0.200 & 22058 & 16904 \\
$63 \times / 0.95$ & 0.23 & 0.270 & 30311 & 23218 \\
$100 \times / 1.30$ & 0.16 & 0.270 & 29441 & 22546 \\
\hline
\end{tabular}

Optics: The instrument was built on the basis of a Fluoval microscope from Carl Zeiss, Jena (Germany). The highly modular design of this instrument allowed realisation of the changes of the optical system described below.

Radiation sources: The function of the system requires that the image field can be irradiated simultaneously by three beams of different character joined into one beam in the last segment of the epifluorescence optical path. These are (Fig. 1): (A) The actinic radiation beam which serves for continuous irradiation of the object to induce fluorescence and achieve its steady state. $(B)$ The pulsed radiation beam from a LED used for excitation of the measured fluorescence signal (further called "measuring pulses"). (C) The beam providing saturating flashes used to excite maximum fluorescence and for fluorescence quenching analysis.

The actinic radiation is generated by a $300 \mathrm{~W}$ superquiet xenon discharge lamp (Hamamatsu Photonics, Shimokanzo, Japan) fed from the electronic power supply EVG 300 DC (Schiederwerk Mbz Telekommunikation, Nürnberg, Germany). The lamp is placed in the standard discharge lamp housing of the Fluoval microscope. The actinic irradiation ( $A$ in Fig. 1) is adjusted in wavelength by colour filters $(I)$, in irradiance by neutral density filters (2), and switched on/off by a computer-controlled mechanical shutter (3) (Uniblitz ${ }^{(\mathbb{B})}$ T132, Vincent Associates, Rochester, NY, USA). It further follows the standard epi-irradiation path $(A$, then $A B C)$ of the microscope. The useful wavelength range of this source is limited by the transmission of the lenses to about $370 \mathrm{~nm}$ at the lower end.

In the section of this light path next to the lens before the dichroic mirror (4), where the beam is parallel, a beam unifying prism (5) has been inserted. The same was done again inside the new radiation path $(B C)$. This allows to couple in two additional radiation sources:

(a) The beam of radiation providing saturating flashes

(C). The radiation source in this case is a $35 \mathrm{~W}$ xenon short-arc lamp [Philips, D2; (6)] produced for car headlamps. The photon flux from this lamp is collimated by an aspheric achromatic lens (7) and a concave mirror (8). The irradiation by this source was adjusted by neutral density filters ( 9 ) between the lens and the mirror. After the mirror, a set of short-pass filters (10) was placed to produce the desired excitation wavelength. Below that, a computer-controlled mechanical (1I) shutter (modified $B A-2$, Carl Zeiss, Jena, Germany) was installed.

(b) Measuring pulses $(B)$ are generated by a LED (12) the radiation of which is collimated by an aspherical lens (13) and combined with the beam $(C)$ by a beam unifying prism (14). For proper operation of the collimating lens it is necessary to cut off the plastic lens tip of the LED and to polish the cut surface. The output of the LED can be regulated by the control electronics. The usable wavelength range of this source is limited by the availability of LEDs to about $380 \mathrm{~nm}$ at the lower end.

The combined beams $(B, C)$, i.e., saturation flashes and measuring pulses, pass once more through a shortpass filter (15) directly above the prism (5) unifying the radiation from the additional sources with that from the conventional epifluorescence source. All radiation sources are adjustable in three dimensions with respect to their condenser lenses in order to produce a proper parallel beam.

The transmittance radiation path of the microscope (D) was not modified. This radiation is generated by a condensed filament tungsten lamp [Narva, $15 \mathrm{~W}$ (10)], passes through colour- and neutral density filters (17), and in the proper position of the switching mirror (18) it enters the path for object irradiation from below. The transmitted irradiation was used only for taking control pictures of the objects measured, which was done with the slow CCD camera (19). Since the camera is much more sensitive than human eye, the transmitted irradiation is dramatically reduced, and taking the snapshots with the camera (19) did not affect the measured objects. 

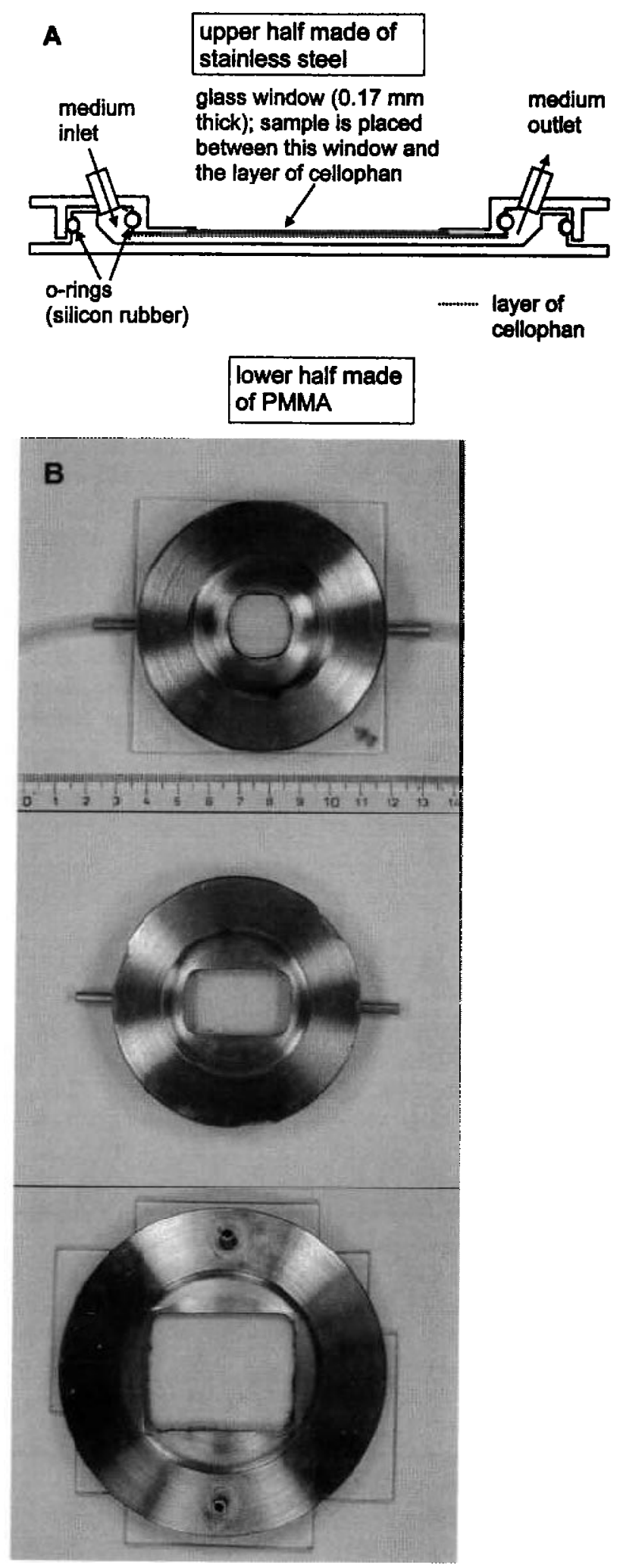

Fig. 2. The thermostated chamber for in vivo measurements under the microscope. $A$ : schematic drawing of the standard version. $B$ : Photographs of the variations of the design. Top: small-window version, middle: version with high cooling compartment, bottom: standard version.
Radiation detection: The object is observed through Zeiss Apochromat objectives with the following technical specifications: $6.3 \times / 0.20,16 \times / 0.40,25 \times / 0.63,40 \times / 0.95$, $63 \times / 0.95,100 \times / 1.30$ oil. Fluorescence collected by one of these lenses $(20)$ enters the detection path $(E)$ at the beginning of which it is separated from reflected excitation radiation by a dichroic mirror (4) and a longpass filter (21). By means of a switching mirror (22) fluorescence can be directed to one of the two highsensitivity b/w Sory ICX259AL CCD chips $(19,23)$. The CCDs have a resolution of $752 \times 582$ effective pixels and are coupled to the computer by an 8-bit A/D-converter and a grabber. This results in maximum of 25 images per $s$ at a $400 \times 300$ pixel resolution.

The CCD chip which is used for the kinetic measurements (23) records the images from a singleMCP image amplifier (Eureca Messtechnik, Koln, Germany) NFBV-25 (24 in Fig. 1), with a red-enhanced $S 25$ photocathode resulting in a sensitivity of $32 \mathrm{~mA} \mathrm{~W}^{-1}$ at $660 \mathrm{~nm}$. The device contains a $25 \mathrm{~mm}$ (effective diameter) MCP with a $P 20$ phosphor screen, and provides an optical resolution of 36 line pairs per $\mathrm{mm}$ at $5 \%$ contrast. The image amplifier is linked to the CCD chip by tapered fibre optics (25). Both the image amplifier and the camera are gated in synchrony with each other and also with the timing and duration of the measuring radiation pulses. The rise time of the gating is shorter than $100 \mathrm{~ns}$, the duration of the gating interval is adjustable from 10 to $250 \mu \mathrm{s}$.

The second CCD (19) is neither gated nor intensified and has been used mainly for capturing transmitted images of measured objects. Since the camera allows also to take fluorescence images with less noise and higher optical resolution than the image amplifier, it is also occasionally used to record still images of fluorescence steady states at either low or high actinic irradiances with integrating times up to $20 \mathrm{~ms}$.

The measurable range of fluorescence wavelengths is limited to about $390 \mathrm{~nm}$ at the lower end by the spectral properties of the measuring radiation and at the upper edge to about $800 \mathrm{~nm}$ by the spectral sensitivity of the image intensifier.

Electronics and software: The control electronics and software of the instrument are based on the commercial FluorCam kinetic imaging fluorimeter from Photon Systems Instruments (Nedbal et al. 2001). As in this system, the total irradiance reaching the CCD during the gating time is recorded, i.e., the fluorescence induced by the measuring radiation plus the background caused by the actinic radiation. The latter is then subtracted using measurements without measuring radiation. These can be done automatically either immediately before every measuring point with measuring radiation, or only in the slower part of the kinetics. In the second case, external spreadsheet software is required to calculate the background signal in the fast part of the kinetics (see also 
"Results and discussion"). To accommodate the specific needs of the present system, the FluorCam electronics and software were modified and supplemented in the following way:

(a) A gating control unit was added to control the image intensifier.

(b) The gain of the MCP can be controlled in addition to the regulation of the CCD camera sensitivity.

(c) The power of the LED measuring pulses can be controlled.

(d) A second trigger pulse output was added to control the actinic irradiation shutter.

Several improvements in the software were carried out to facilitate the analysis of signals that are weaker and noisier than the signals from the FluorCam operating on the macroscopic scale.

Determination of irradiation at the sample level: The diameters of the image fields seen by the CCD cameras with the different objectives used were measured with a fluorescing object micrometer. The diameters of the fields irradiated by the actinic and saturating sources at the sample level are larger and were assessed in the following way: The maximum available irradiance (Table 1) was applied through the objective to a Mnium leaf (which has only one layer of cells) for $5 \mathrm{~min}$. In contrast to a normal measurement (for irradiances see Fig. legends), this produced a persistent photoinhibition in the irradiated area. The image of the corresponding change in fluorescence yield was afterwards recorded through a lower-magnification objective and its diameter was determined by comparison with the object micrometer. In the lowest magnification $(6.3 \times)$, the irradiated field was large enough $(2.9 \mathrm{~mm})$ to be measured directly with a calliper.

These measurements were used to determine the irradiances on the sample level for the various lenses and the three types of beam used (see Table 1). The procedure was as follows: Actinic radiation was passed through the $6.3 \times$ objective producing a reasonably homogeneous irradiation on a circular area of $50-\mathrm{mm}$ diameter. The average irradiance of the field was measured by a conventional light meter with a quantum proportional response. The ratio of the areas $\left(50.0^{2}: 2.9^{2}\right)$ allowed the calculation of irradiance on the sample level for this lens. Since the other lenses did not provide a homogeneous enough field outside the focus plane, the total amount of radiation passing through the individual objectives was measured with the sensor directly applied to the front lens. The irradiance on the sample level of the tested objective was calculated as follows:

$$
\operatorname{irr}=(\text { irradiance of } 6.3 \times \text { objective }) \cdot \frac{\frac{\text { (radiation throughput of tested objective) }}{\text { (irrad. field of tested objective) }}}{\frac{\text { (radiation throughput of } 6.3 \times \text { objective) }}{(\text { irrad. field of } 6.3 \times \text { objective })}}
$$

Measuring conditions: For all measurements, the following settings of the microscope were used. These can be modified to excite and measure different types of fluorescence (e.g., fluorescence of phycobilisomes and fluorescent dyes), as long as these are within the maximum ranges specified in the "Optics" section. The object numbers/letters in the following paragraph refer to Fig. 1.

- Measuring flashes (B): LED Nichia (Tokushima, Japan) $N S P B 500 S, \lambda_{\max }=470 \mathrm{~nm}$, optical output power $6 \mathrm{~mW}$. The radiation of this diode passed through the same custom-made heat and $490 \mathrm{~nm}$ short pass filters (15) as the saturating radiation to remove the near infrared radiation produced by blue LEDs in addition to their main emission peak. Measuring irradiance was attenuated to $\max .0 .2 \mu \mathrm{mol} \mathrm{m} \mathrm{m}^{-2} \mathrm{~s}^{-1}$ on the sample level for $F_{0}$ measurements; the exact irradiance is given with the individual examples presented.

- Saturating radiation (C): a custom-made $490 \mathrm{~nm}$ shortpass filter (10) and a Schott (Mainz, Germany) $B G 18$ filter (15) were used to obtain blue radiation of $350-450 \mathrm{~nm}$, which was lowered to about $1000 \mu \mathrm{mol}$ $\mathrm{m}^{-2} \mathrm{~s}^{-1}$ on the sample level by neutral density filters $(8)$.
The maximum irradiances on the observed object that are achievable with this set of filters are listed in Table 1.

- Actinic radiation $(A)$ : Schott $b 421 \mathrm{~g}$ and $b 228 \mathrm{~g}$ filters and two custom-made $490 \mathrm{~nm}$ short pass filters $(I)$ were used to obtain blue radiation of $350-450 \mathrm{~nm}$ (for max. achievable irradiances see Table 1). Irradiance was attenuated by neutral density filters (2) to the value stated in the individual examples.

- Transmittant radiation $(D)$ : A combination of shortpass, long-pass, and neutral density filters (17) was used to produce $600-650 \mathrm{~nm}$ radiation with an irradiance of less than $1 \mu \mathrm{mol} \mathrm{m} \mathrm{m}^{-2} \mathrm{~s}^{-1}$ on the sample level.

- Fluorescence detection: a dichroic mirror (4) with $510 \mathrm{~nm}$ edge wavelength and a Zeiss 0263 long-pass filter (21) were used to observe fluorescence and transmitted radiation above $600 \mathrm{~nm}$.

Measuring chamber: Measurements with diverse photosynthetic - especially aquatic - organisms have demonstrated the general necessity to use a measuring chamber for controlling conditions during the measurement. Without a measuring chamber, i.e., under a standard cover glass, samples dry out within a short time, gas exchange conditions cannot be controlled, and irradiation 
also leads to rise of the sample temperature, making measurements under a cover glass irreproducible.

To ensure defined physiological conditions for the microscopic objects during the measurement as well as reproducibility of results, temperature and composition of the medium and of the dissolved gas mixture have to be under control. We did not find any commercially available device providing such conditions during observations under a microscope. We therefore constructed several variants of the measuring chamber the design principle of which is drawn in Fig. $2 A$. In short it can be described as follows:

The object (algal cells, moss leaflets, leaves of water plants, etc.) is kept appressed to the inner surface of the glass window by soft cellophane, which is stretched over the top surface of the chamber and pulled tight by an $\mathrm{O}-$ ring. Temperature-controlled medium is pumped through the chamber using a centrifugal pump. When using aqueous media, a flow rate of about $3.33 \mathrm{~cm}^{3} \mathrm{~s}^{-1}$ was used. This was found sufficient for keeping a constant temperature of the chamber even if the room temperature was by $10^{\circ} \mathrm{C}$ different: Under these conditions, the outflow of the chamber differed by $1{ }^{\circ} \mathrm{C}$ from the temperature inside the thermostat. Inside the thermostat, the medium was enriched with the desired gas mixture by bubbling the gas through the medium. In all measurements presented here, the medium was saturated with air.

The individual variants of the chamber (Fig. $2 B$ ) vary mainly $(l)$ in their diameter and (2) in the height of the cooling compartment. Chambers with small diameter

\section{Results and discussion}

Performance of the measuring system: The earlier published systems detect only individual fluorescence parameters (e.g., Oxborough and Baker 1997b) or record fluorescence kinetics in a single-point (Schreiber 1998). In contrast, the new instrument enables to display complete fluorescence induction curves, including the recording of $F_{0}$ and a quenching analysis with saturating flashes. The use of pulse-modulated excitation radiation allowing the measurement of fluorescence yield brings along all the advantages inherent to this approach. The most important is clean separation of the measured signal from background fluorescence excited by other beams applied to the sample. In this way, the "relative fluorescence" in Figs. 5-10 represents the true fluorescence quantum yield, i.e., net signal of the measuring radiation after background subtraction. The maximum time resolution of $40-80 \mathrm{~ms}$ achieved in our device is sufficient for reliable recording of transients of medium velocity which display most of the physiologically relevant characteristics of the induction curves and other kinetic responses to changes of external factors (e.g., oscillations). The CCD camera imposes the $40 \mathrm{~ms}$ limit have the advantage of lesser vibrations of the objects held by the cellophane, which is particularly desirable for records at maximum magnification (100x objective). Larger chambers, such as the basic model (Fig. 2), greatly facilitate the screening of larger samples to find an object of choice (e.g., to find a sporangium in a large number of non-fertile algae filaments). Chambers with a minimum depth of the medium layer yield better pictures taken in transmitted radiation. An increased volume of the cooling compartment provides better temperature control, especially when air is used instead of aqueous media.

Plants: The marine filamentous brown alga Ectocarpus siliculosus (Dillwyn) Lyngbye (strain Port Aransas) was kindly provided by D.G. Müller and I. Maier (Universität Konstanz). The chlorococcal freshwater green alga Scenedesmus quadricauda (Turp.) Bréb (strain Greifswald 15) was grown permanently for various experiments in the Institute of Microbiology in Treboñ; the stock culture of the strain was obtained from the culture collection of the Botanical Institute of the Academy of Sciences of the Czech Republic in Trebon. The green alga Micrasterias crux-melitensis (Ehrenb.) Hass. was kindly provided by A. Couté (Museum National d'Histoire Naturelle, Paris). The moss Mnium cuspidatum (Hedw.) was collected in a forest near Tłebon (Czech Republic). The submerged freshwater macrophyte Elodea canadensis Michx. was collected from a pond near Marl (Westphalia, Germany). Hibiscus sp. was a pot plant grown in the laboratory.

of time resolution. At present, a faster reading with the same sensitivity is not easy to realise. Therefore, fast transients in the millisecond and sub-millisecond time range are outside the potential of the instrument. The $80 \mathrm{~ms}$ limit applies to measurements in which the background is being subtracted from the value measured with the measuring pulse on. This is the mode most often used for convenience. If it is desirable to record a certain part of the transient curve with a better time resolution, the $40 \mathrm{~ms}$ intervals of measuring flashes can be applied and the fluorescence response can be measured during each flash without subtraction of the background values. In this case, the relation of background signal to measuring signal has to be quantified in a later (slower) part of the kinetics using external spreadsheet software.

Tests with diverse photosynthetic materials showed that measurements are possible with a wide range of objectives, i.e., from the minimum view field of $100 \times 73 \mu \mathrm{m}$ in the $100 \times$ objective where one pixel represents $0.25 \mu \mathrm{m}$, to the maximum view field of $1600 \times 1200 \mu \mathrm{m}$ in the $6.3 \times$ objective (i.e., 1 pixel $=$ $4 \mu \mathrm{m})$. As shown in Table 1 , the power of the objective 
used for imaging has a decisive influence on the sample irradiance, and the numerical aperture on the fluorescence collection efficiency. These properties are important limiting factors in the performance of our FKM because its sensitivity limit depends on the capacity of the electronic equipment to detect the weak signal excited by the measuring pulses. Hence, the factors limiting its performance differ principally according to the power of the objective used for imaging.

At the lower end of magnifications, the instrument is capable of measuring objects on a semi-macroscopic scale (measurements with Hibiscus in Fig. 10; biological details are discussed later), i.e., directly the order of magnitude below the size which can still be analysed with the macroscopic FluorCam (Nedbal et al. 2001). In the macroscopic device the technical problems are posed mainly by the request of sufficient irradiance of the measured objects in both the actinic and measuring range. In our case, thanks to high concentration of the photon fluxes under the objectives, there is no problem to achieve saturation with continuous actinic irradiation and flashes (see Table 1). However, the power of measuring flashes generated by a commercially produced highoutput blue LED is still limiting, particularly with low power objectives. Such lenses have much lower apertures than high-power lenses, and the efficiency of the capture of fluorescence increases with square of the aperture. Thus, the $40 \times$ lens with an aperture of 0.95 has a 22.5 times higher efficiency in capturing fluorescence than the $6.3 \times$ lens with an aperture of 0.2 . For getting a reasonable signal with a low-power objective, one has to use long integration times ( 100 or even $250 \mu$ s). This leads to degradation of the signal/background ratio: with the same total measuring and actinic irradiances, an $\mathrm{x}$ times prolongation of the measuring flash duration yields a $1 / x$ times lower (measuring signal)/(actinic background) ratio. This may be principally solved using stronger measuring flashes. Since the $F_{0}$ measuring radiation at this magnification is presently only around $0.006 \mu \mathrm{mol} \mathrm{m}^{-2} \mathrm{~s}^{-1}$ with $100 \mu \mathrm{s}$ flashes, it is possible to increase the measuring irradiance 10-30 times without reaching the actinic irradiance. Since the pulse duration has to be exactly defined and adjustable, the pulse amplitude and pulse delay precisely reproducing xenon flash lamps do not present an alternative to the LEDs used. This was tested also in the FKM. Hence, only laser flashes promise a solution, and this is currently under construction in our system.

At the high end of magnifications, measurements with Ectocarpus (Fig. 7) and Elodea (Fig. 9) showed that the system might measure the fluorescence yield in individual chloroplasts. In this range the power of measuring flashes is not limiting. The presently used measuring irradiance is ca. $0.3 \mu \mathrm{mol} \mathrm{m} \mathrm{m}^{-2} \mathrm{~s}^{-1}$ (with $100 \mu \mathrm{s}$ flashes) with the $63 \times$ and $100 \times$ objectives, which for most plants is just below the threshold of actinic effects.
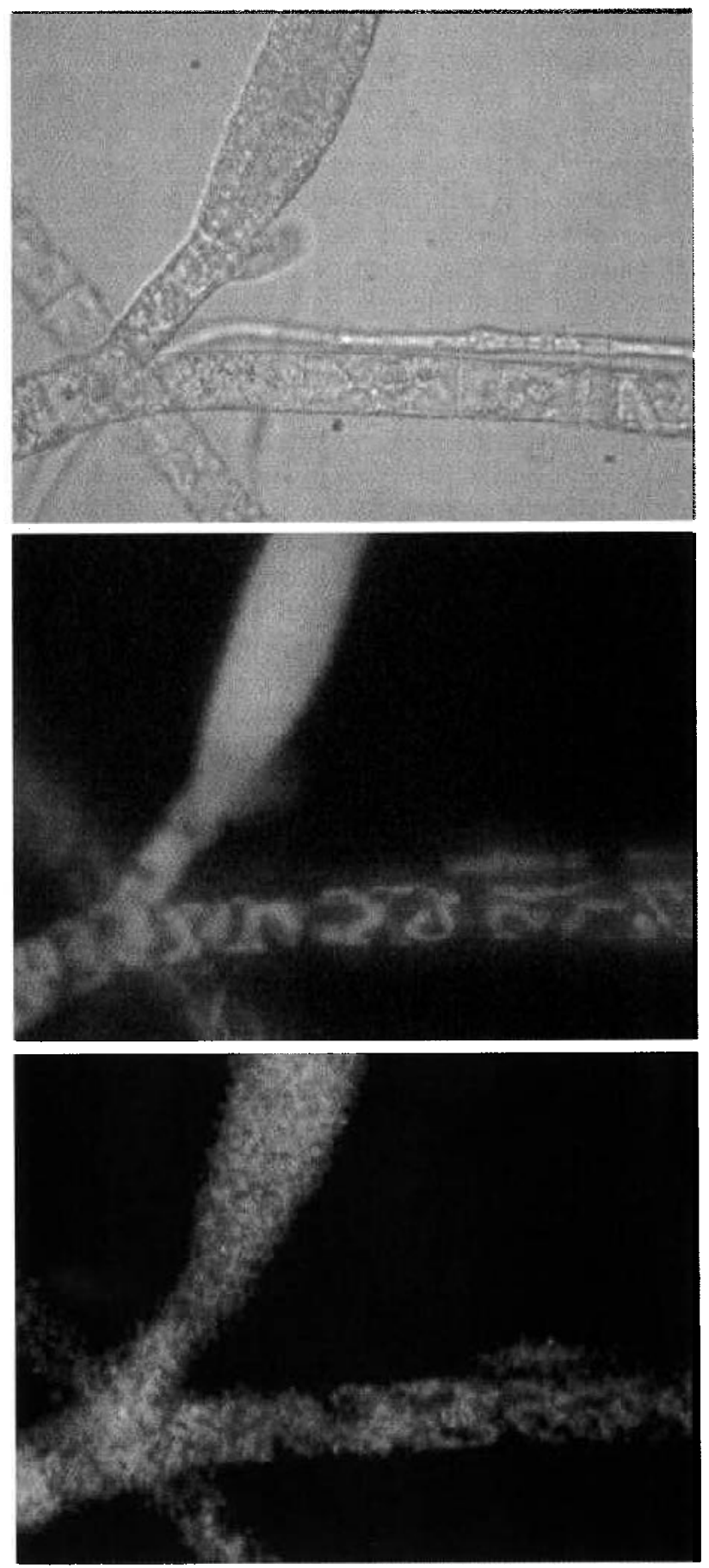

Fig. 3. MCP vs. CCD: Pictures of Ectocarpus siliculosus (somatic cells and sporangium) taken with the slow nonamplified $C C D$ and the fast MCP-intensified CCD. The width of the images represents $240 \mu \mathrm{m}$. Top: transmitted picture taken with the slow CCD. Middle: Image of steady state chlorophyll fluorescence at an irradiance of $190 \mu \mathrm{mol} \mathrm{m} \mathrm{m}^{-2} \mathrm{~s}^{-1}$ taken with the slow CCD, integrating time $20 \mathrm{~ms}$. Bottom: Image of steady state chlorophyll fluorescence taken with the fast $\mathrm{CCD}$, integrating time and measuring flash duration $30 \mu \mathrm{s}$, measured with measuring flashes, average of 8 frames. 
At this magnification, the main question is that the records contain already a significant noise. This partially originates from the statistic variations of the number of photons reaching the image amplifier. Therefore, using the same measuring irradiance, the reduction of noise (e.g., in the fluorescence kinetics of a chloroplast) by using the $100 \times$ objective instead of the $40 \times$ one is much less than one would expect from the increase of the number of pixels that can be used for averaging. This is demonstrated by comparing the curves in Fig. $9 B$ and in its inset. The effect is worsened by the characteristics of image amplifier that must amplify the fluorescence signal to the level recordable by the camera. Image amplifiers have a significant noise, which originates mainly from two sources. On the one hand, they produce a significant dark noise, which is especially high in the red-enhanced photocathodes necessary for detecting Chl fluorescence.

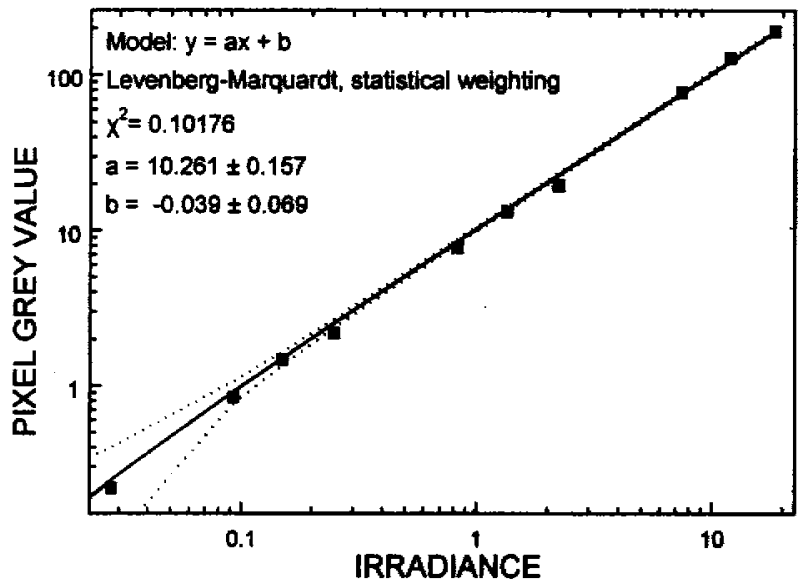

Fig. 4. Linearity of the detection system (fast CCD with image amplifier). Fluorescence of chlorophyll $a$ solution in oil was measured at different actinic irradiances. Squares: individual values, straight line: fitted function, dotted lines: $95 \%$ confidence intervals.

The dark noise can be removed by using shorter gating times and correspondingly stronger pulses of measuring radiation. On the other hand, image amplifiers have fairly low quantum efficiency in the red region; even the redenhanced photocathode used in our system has only around $6 \%$ quantum efficiency at $700 \mathrm{~nm}$. This further decreases the signal/noise ratio if the noise from the statistic variation of the fluorescence photon flux contributes a significant proportion to the total noise, as mentioned above. This noise problem could be alleviated only by replacing the intensified CCD camera with a nonintensified $C C D$ of extremely high sensitivity, since CCD chips can achieve quantum yields of over $80 \%$ at $700 \mathrm{~nm}$. Using such a sensitive CCD with additional cooling to reduce the dark noise and with 12-bit resolution to increase the dynamic range, the sensitivity of the present system might be reached with a better signal/noise ratio. Additionally, image amplifiers offer a much lower spatial resolution than CCD chips (Fig. 3), leading to blurred images. This is caused mainly by the characteristics of phosphor screen, which yields a ten times lower resolution than one would expect from the size of the individual micro-channels below. However, the technical specifications of CCD chips present on the market show that the available sensitivity is not yet high enough to enable measurements with non-actinic measuring radiation which is necessary for $F_{0}$ determination. Therefore, at the moment the use of bare CCDs is limited to cases where long integration times can be used to compensate the low sensitivity, i.e., to capture still pictures of fluorescence. This is done in our system with the slow $C C D$, and is the only way of imaging applied in the system of Oxborough and Baker (1997a, b).

Tests with $\mathrm{Chl}$ solutions in oil showed that the standard errors of the distribution of irradiance (measured as pixel grey values) are as follows: Actinic radiation $<5 \%$, measuring radiation around $6 \%$ (exact value depending on LED), saturating radiation $11 \%$. The system was optimised for homogeneity of the actinic radiation; a non-homogeneity in the saturating flashes

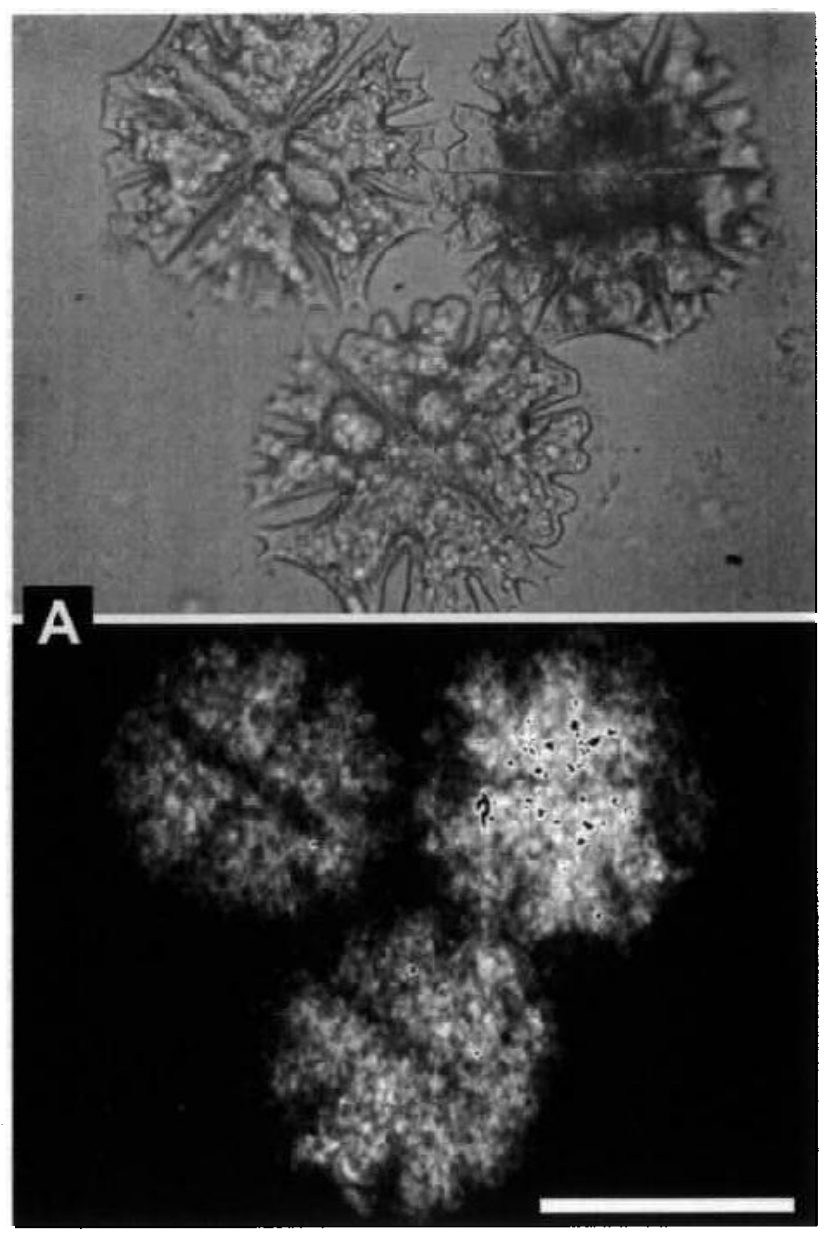




\section{H. KÜPPER et al.}
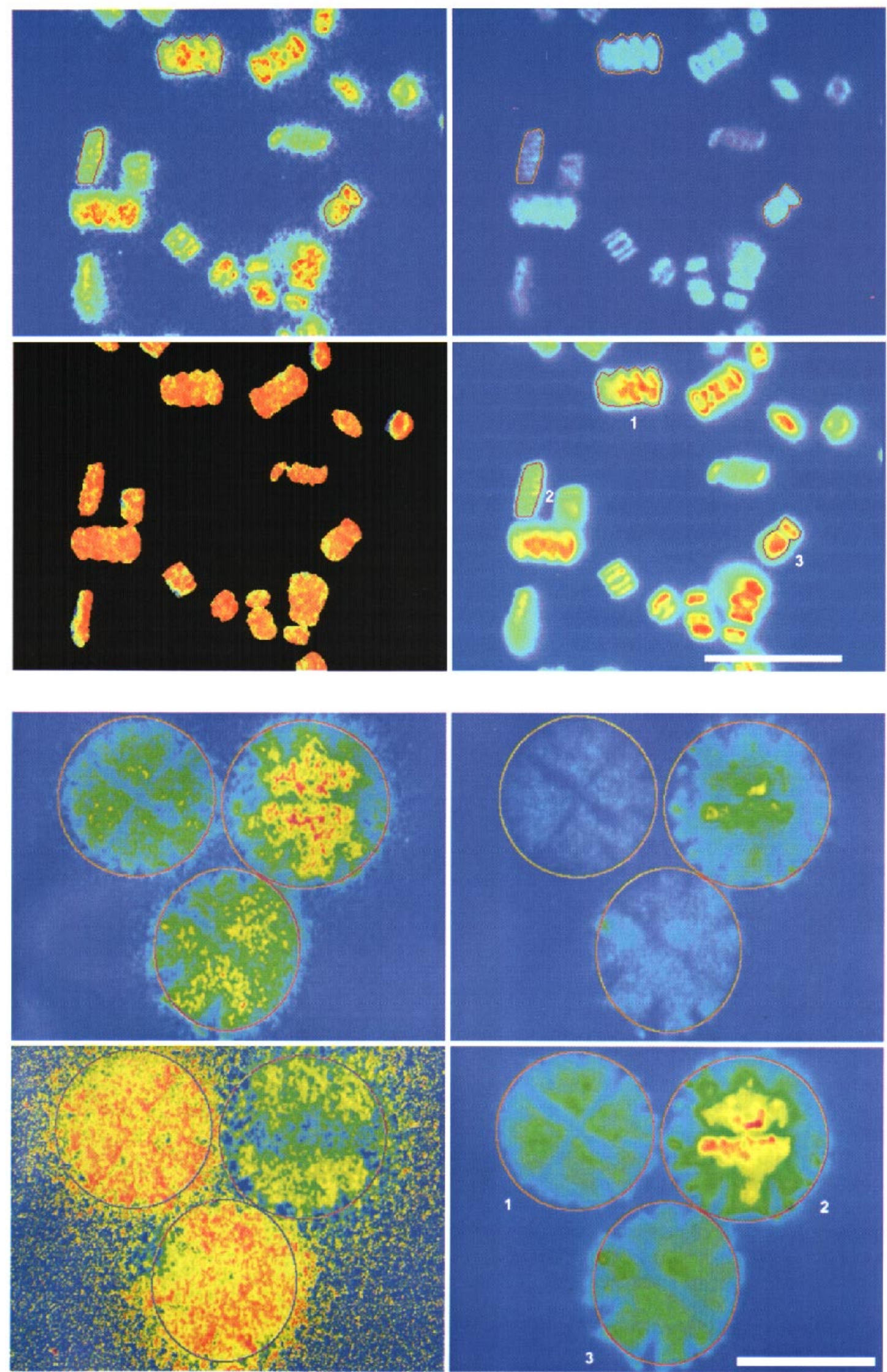


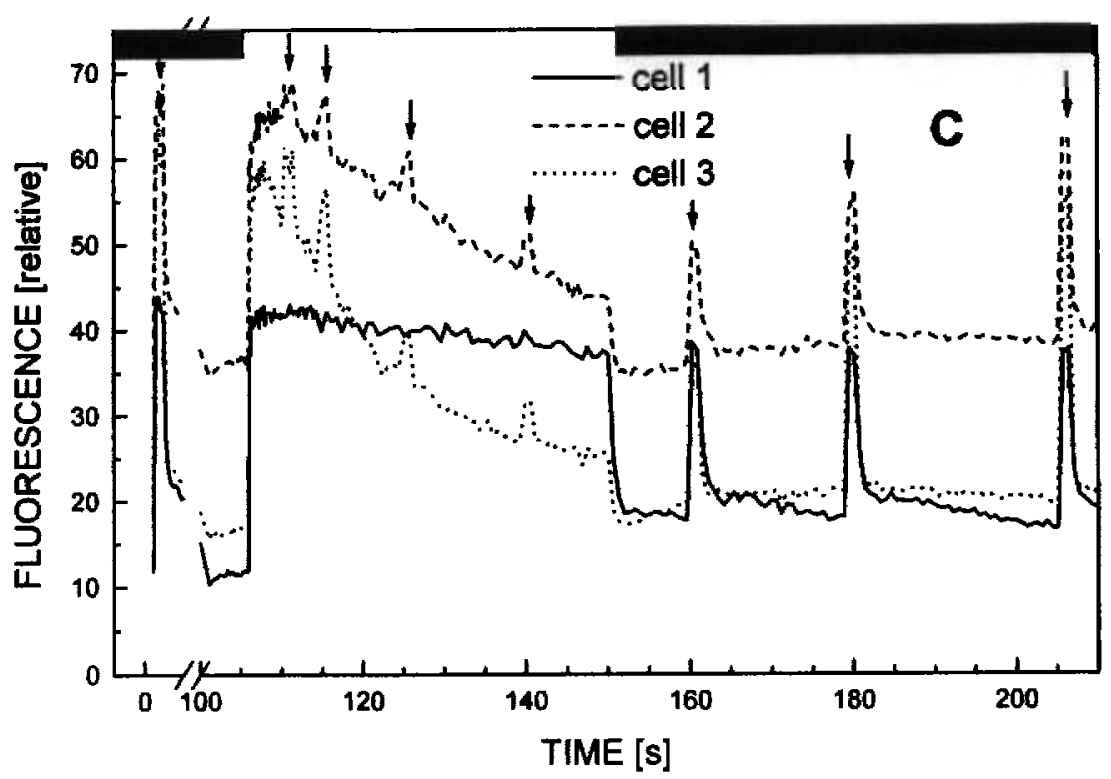

Fig. 5. Chlorophyll a fluorescence kinetic microscopy of a suspension of Micrasterias crux-melitensis. Measurement with the $40 \times$ objective at $25^{\circ} \mathrm{C}$, with $c a .60 \mathrm{~s}$ dark adaptation before measurement and $110 \mu \mathrm{mol} \mathrm{m} \mathrm{m}^{-2} \mathrm{~s}^{-1}$ actinic irradiance from 105 to $145 \mathrm{~s}$ after start of measurement. $A$ (p. 563): Snapshot images of the objects [taken with the slow CCD (top) or the fast CCD (bottom) during the measurement $1 \mathrm{~s}$ after the onset of actinic irradiation]; the bar represents $50 \mu \mathrm{m} . B$ (p. 564 , top pane/s): False colour images of the measurement and objects selected for the curves; the bar represents $50 \mu \mathrm{m}$. Top left: $\mathrm{F}_{\mathrm{M}} ;$ top right: $\mathrm{F}_{0} ;$ bottom left: $\mathrm{F}_{\mathrm{v}} / \mathrm{F}_{\mathrm{M}}$ with the spectrum colour scale assignment $0=$ blue and $1=$ red; bottom right: average fluorescence of entire measurement. $C$ : Kinetic fluorescence curves of selected cells. The arrows indicate the positions of saturating flashes. The "relative fluorescence" in all figures represents the true fluorescence quantum yield, i.e., net signal of the measuring radiation after background subtraction.

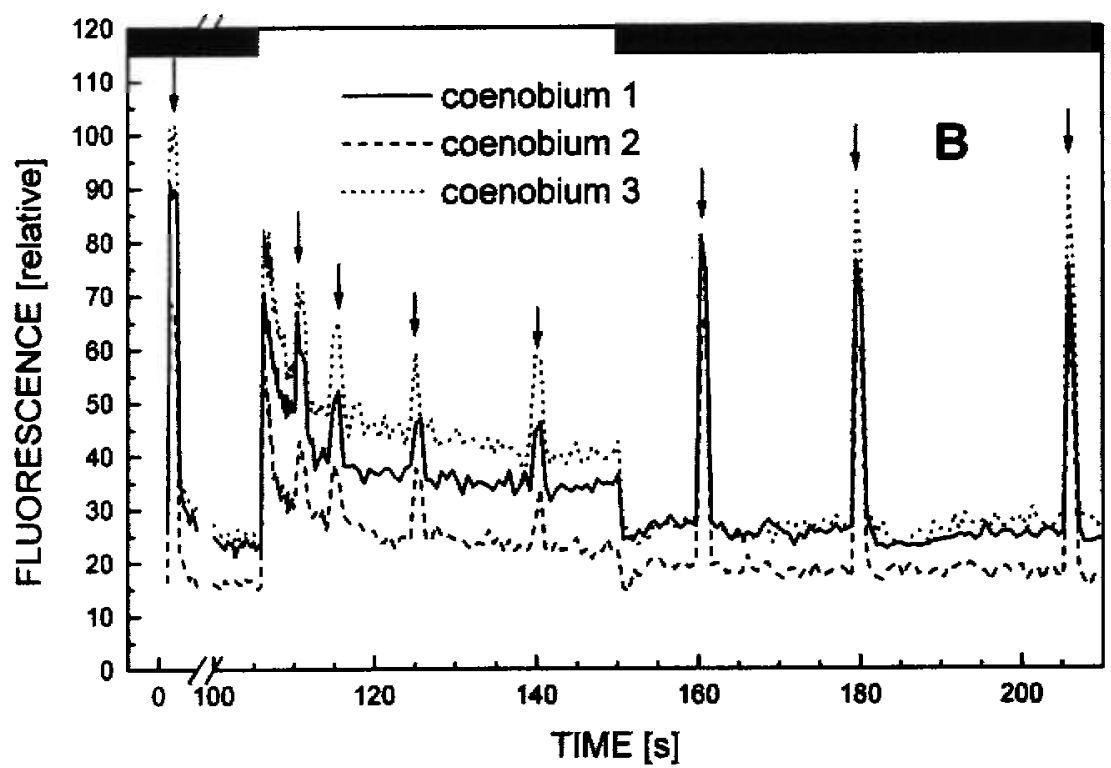

Fig. 6. Chlorophyll a fluorescence kinetic microscopy of a suspension of Scenedesmus quadricauda immobilised with $1 \%$ ultra lowgelling $\left(10^{\circ} \mathrm{C}\right)$ agarose. Measurement with the $40 \times$ objective at $28^{\circ} \mathrm{C}$, with ca. $60 \mathrm{~s}$ dark adaptation before measurement and $100 \mu \mathrm{mol} \mathrm{m} \mathrm{m}^{-2} \mathrm{~s}^{-1}$ actinic irradiance from 105 to $145 \mathrm{~s}$ after start of measurement. $A$ (p. 564, bottom panels): False colour images of the measurement. Top left: $\mathrm{F}_{\mathrm{M}}$; top right: $\mathrm{F}_{0}$; bottom left: $\mathrm{F}_{\mathrm{v}} / \mathrm{F}_{\mathrm{M}}$ with the assignment $0=$ blue and $0.9=$ red; bottom right: average fluorescence of entire measurement and objects selected for the curves. $B$ : Fluorescence kinetics of selected cells. The arrows indicate the positions of saturating flashes. 


\section{H. KÜPPER et al.}
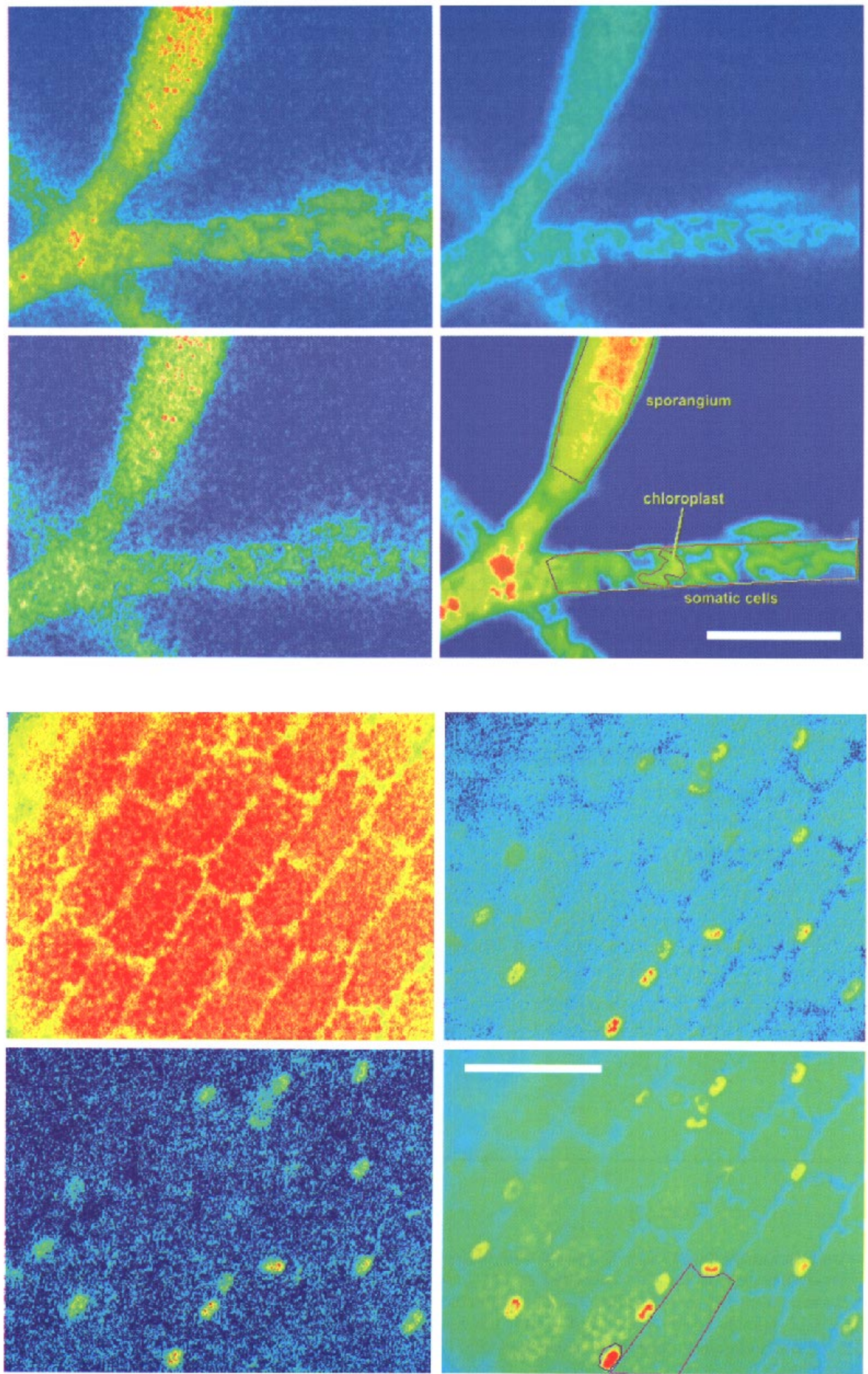


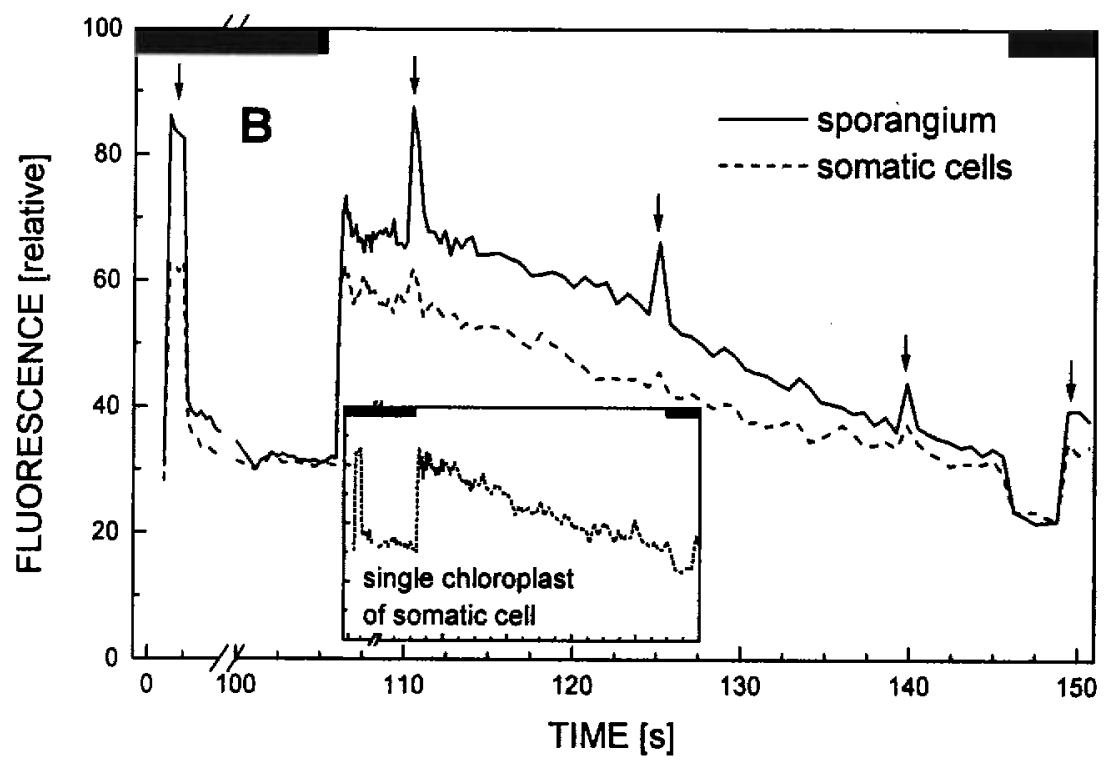

Fig. 7. Chlorophyll a fluorescence kinetic microscopy of Ectocarpus siliculosus filaments (same type of measurement as used for Fig. 3). Measurement with the $40 \times$ objective at $15^{\circ} \mathrm{C}$, with ca. $60 \mathrm{~s}$ dark adaptation before measurement and $190 \mu \mathrm{mol} \mathrm{m} \mathrm{m}^{-2} \mathrm{~s}^{-1}$ actinic irradiance from 105 to $145 \mathrm{~s}$ after start of the measurement. $A$ (p. 566, top panels): False colour images of the measurement. The bar represents $50 \mu \mathrm{m}$. Top left: $\mathrm{F}_{\mathrm{M}} ;$ top right: $\mathrm{F}_{0} ;$ bottom left: $\mathrm{F}_{\mathrm{v}} ;$ bottom right: average fluorescence of entire measurement and objects selected for the curves. $B$ : Fluorescence kinetics of somatic cells and a plurilocular sporangium, normalised to equal $F_{0}$ for easier comparison. The arrows indicate the positions of saturating flashes.

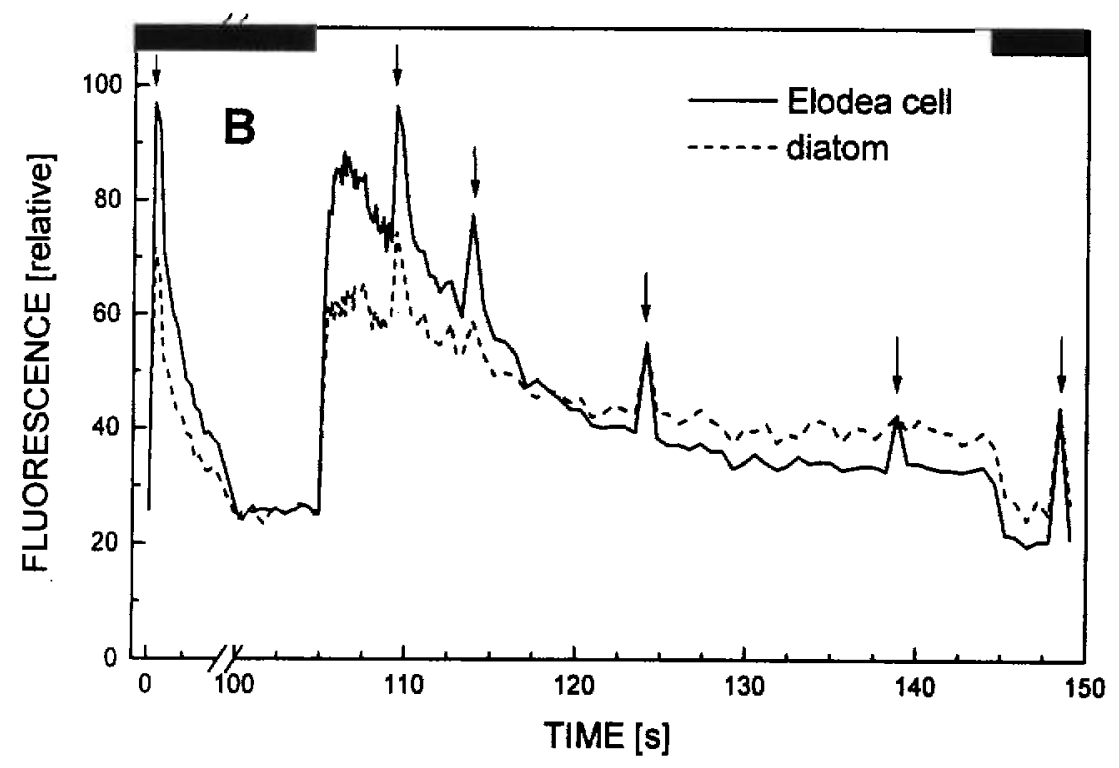

Fig. 8. Chlorophyll $a$ fluorescence kinetic microscopy of an Elodea canadensis leaf with epiphytic diatoms. Measured with the $40 \times$ objective at $20^{\circ} \mathrm{C}$, with $\mathrm{ca} .60 \mathrm{~s}$ dark adaptation before measurement and $56 \mu \mathrm{mol} \mathrm{m}^{-2} \mathrm{~s}^{-1}$ actinic irradiance from 105 to $145 \mathrm{~s}$ after start of measurement. $A$ (p. 566, bottom panels): False colour images of the measurement. The bar represents $50 \mu \mathrm{m}$. Top left: $\mathrm{F}_{\mathrm{v}} / \mathrm{F}_{\mathrm{M}}$ with the assignment 0.2 = blue, $0.8=$ red; top right: $\mathrm{F}_{\mathrm{g}}$; bottom left: $\mathrm{F}_{\mathrm{s}}-\mathrm{F}_{0}$; bottom right: average fluorescence of entire measurement and objects selected for the curves. $B$ : Fluorescence kinetic curves of selected Elodea cells and diatoms, normalised to equal $F_{0}$ for easier comparison. The arrows indicate the positions of saturating flashes. 
H. KÜPPER et al.
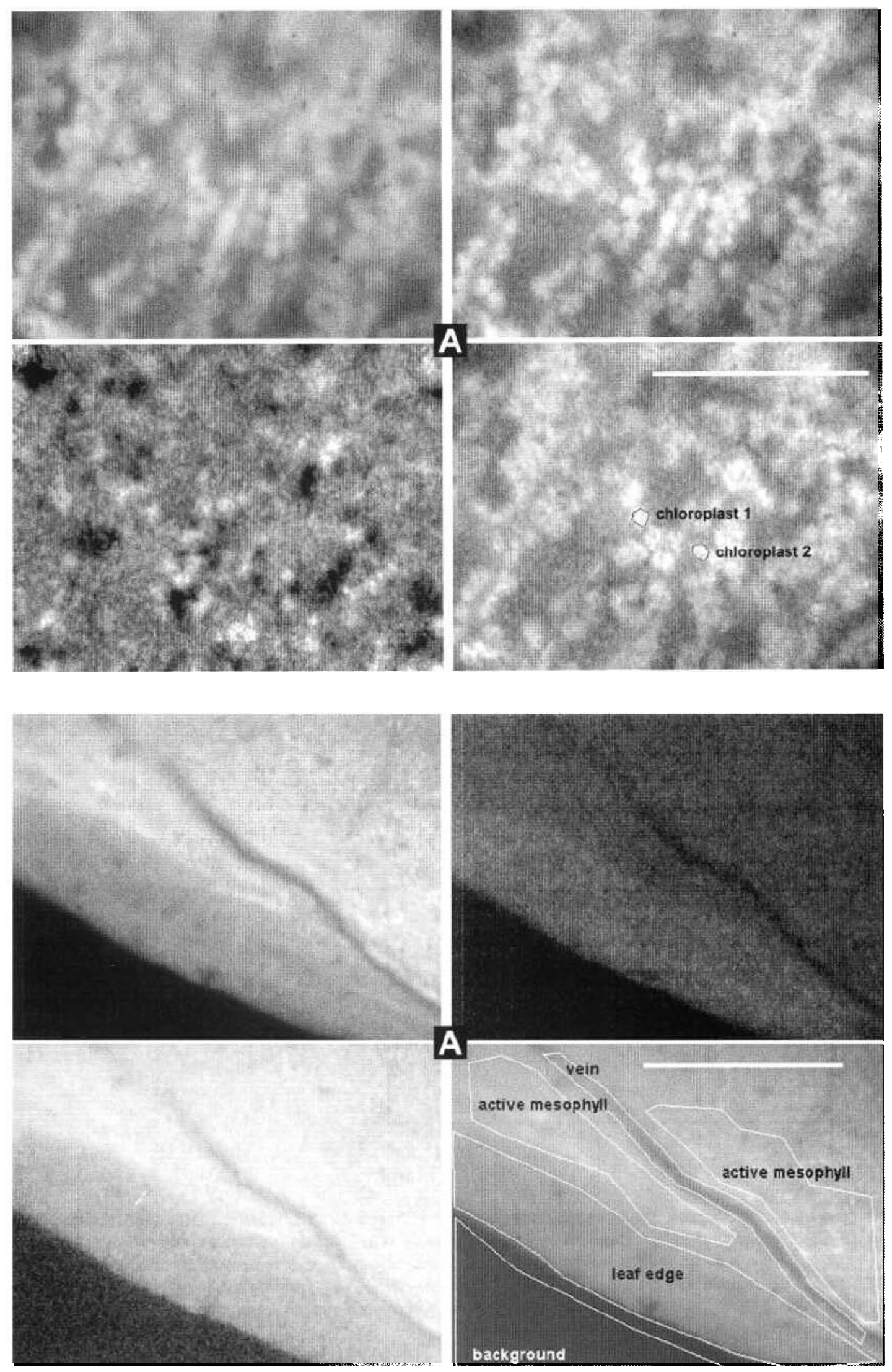


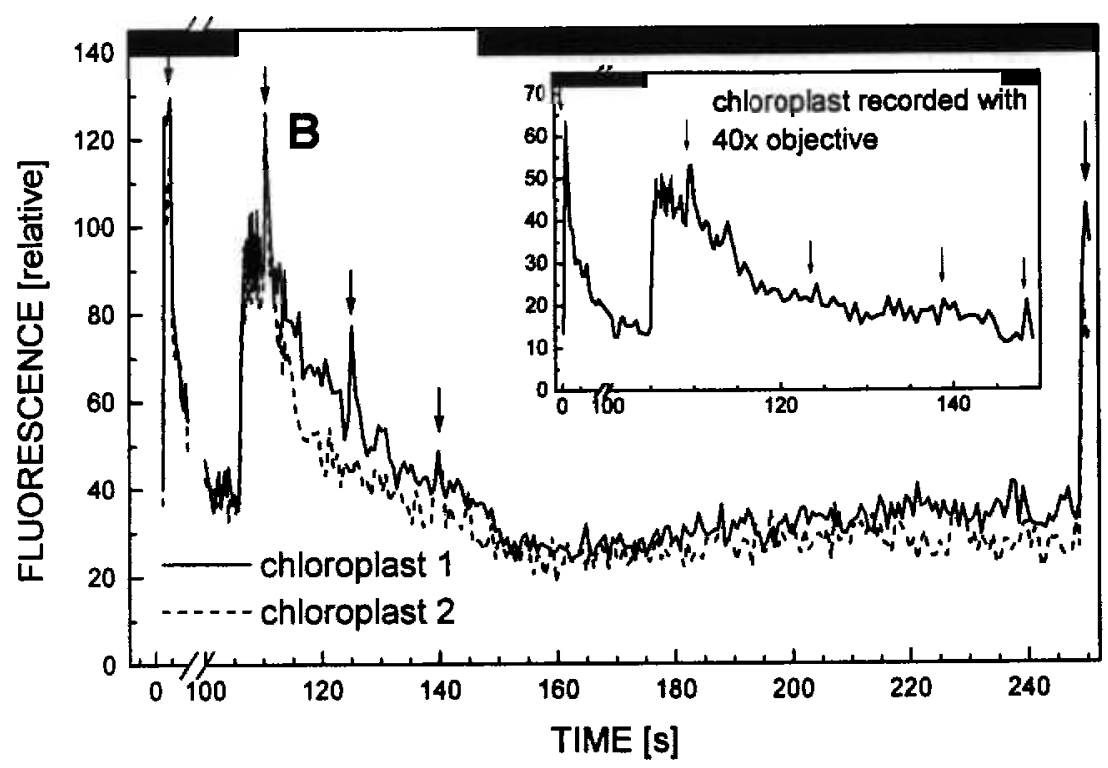

Fig. 9. Chlorophyll a fluorescence kinetic microscopy of an Elodea canadensis leaf measured with the $63 \times$ objective at $20^{\circ} \mathrm{C}$, with ca. $60 \mathrm{~s}$ dark adaptation before measurement and $46 \mu \mathrm{mol} \mathrm{m}^{-2} \mathrm{~s}^{-1}$ actinic irradiance from 105 to $145 \mathrm{~s}$ after start of measurement. $A$ (p. 568 , top panels): Images of the measurement. The bar represents $50 \mu \mathrm{m}$. Top left: $\mathrm{F}_{0}$, i.e., $\mathrm{F}_{0}$ immediately after switching off the actinic radiation; top right: $\mathrm{F}_{0} 100 \mathrm{~s}$ after end of actinic radiation, further called $\mathrm{F}_{0}$ "; bottom left: $\mathrm{F}_{0}$ "' $\mathrm{F}_{0}{ }^{\prime}$ with the greyscale assignment $1=$ black and $2.5=$ white; bottom right: $\mathrm{F}_{0}$ with chloroplasts selected for the curves. $B$ : Fluorescence kinetics of selected chloroplasts, normalised to equal $F_{0}$ for easier comparison. The arrows indicate the positions of saturating flashes. Small inset: Measurement of a chloroplast with the $40 \times$ objective (same recording as used for Fig. 10).

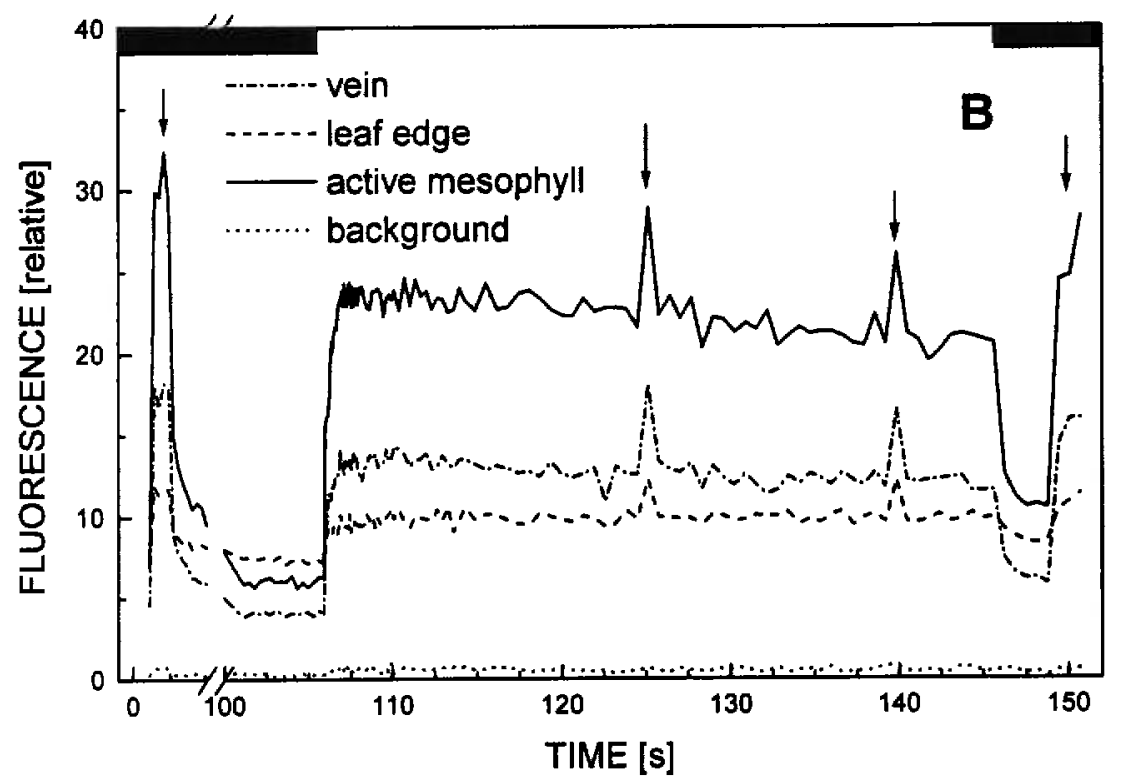

Fig. 10. Chlorophyll a fluorescence kinetic microscopy of the lower side of a Hibiscus leaf measured with the $6.3 \times$ objective at $20^{\circ} \mathrm{C}$, with ca. $60 \mathrm{~s}$ dark adaptation before measurement and $85 \mu \mathrm{mol} \mathrm{m} \mathrm{m}^{-2} \mathrm{~s}^{-1}$ actinic irradiance from 105 to $145 \mathrm{~s}$ after start of measurement. $A$ (p. 568, bottom panels): Images of the measurement. The bar represents $500 \mu \mathrm{m}$. Top left: $\mathrm{F}_{\mathrm{M}} ;$ top right: $\mathrm{F}_{0} ;$ bottom left: $\mathrm{F}_{\mathrm{v}} / \mathrm{F}_{\mathrm{M}}$ with the greyscale assignment $0=$ black and $1=$ white; bottom right: average fluorescence of the entire measurement and objects selected for the curves. $B$ : Fluorescence kinetic curves of selected leaf areas. The arrows indicate the positions of saturating flashes. 
does not affect the results as long as the flash is saturating even at the weakest point of the radiation field. A slight non-homogeneity in the measuring radiation field does not affect the shape of the curves as well, since this radiation is per se non-actinic. The variability of the radiation output (e.g., by instability of the arc or the power supply) of all radiation sources was smaller than $1 \%$ if the discharge lamps had been started at least 30 min before measurement. Linearity of the fluorescence detection $(\mathrm{MCP}+\mathrm{CCD})$ was almost perfect over three orders of magnitude (Fig. 4).

Each basic record contains the programmed number of images comprising 120000 pixels each, in which any area of interest can be selected to display the full kinetics of fluorescence of the objects chosen. The minimum size of the object that can yield a reasonable signal-to-noise ratio of the curves depends on the noise level of the basic record. With records of good quality (as represented, e.g., on Fig. 8) it is around 20 pixels. The maximum number of areas that can be simultaneously analysed by the software (256 with the manual, 6000 with the automatic selection mode) practically always surpasses the number that is reasonable from a physiological point of view.

Examples of photosynthetic heterogeneity on the microscopic scale: The range of objects in which fluorescence kinetics can be effectively recorded by the microscope with the present parameters is best characterised by several examples.

In the highest magnification range, records of twodimensional kinetics of fluorescence yield in individual chloroplasts or their parts can be obtained. This applies particularly to large algae cells with only one chloroplast, such as Micrasterias. Fig. 5 is a record (objective $40 \times$ ) of three Micrasterias crux-melitensis cells. Fig. $5 \mathrm{~A}$ shows the picture taken in transmitted radiation with the slow CCD (left) and one of the frames from the fluorescence kinetic record in the same cells (right). The latter has been taken in the first second of actinic irradiation by the amplified measuring camera under conditions specified in the legend to Fig. 5. Fig. $5 B$ shows false colour maps of the values of fluorescence parameters in different intervals of the fluorescence induction. These are the average of fluorescence yield $\left(\Phi_{F}\right)$ during the measurement (bottom right panel), $\Phi_{F}$ in the period when the cells are not irradiated $\left(\mathrm{F}_{0}\right.$, top right), and $\Phi_{\mathrm{F}}$ during a saturating flash excitation $\left(\mathrm{F}_{\mathrm{M}}\right.$, top left). An image representing in a false-colour scale the map of values resulting from a pixel by pixel performed operation $\left(\mathrm{F}_{\mathrm{M}}-\mathrm{F}_{0}\right) / \mathrm{F}_{\mathrm{M}}$, is shown in the bottom left panel of Fig. $5 B$. This image, therefore, displays the distribution of values characterising the photochemical efficiency of PS2. As the numerical scale referring to the palette of false colours (not shown) indicates, the values of this fraction lie between 0.30 and 0.78 . If the whole window is selected for analysis as in the present case, the
$\left(F_{M}-F_{0}\right) / F_{M}$ operation is also performed on the background. This causes the coloured veil around the objects. For this reason we prefer to perform the last operation only for selected areas, confined to the images of the cells (see Figs. 6 and 7).

Either the $b / w$ image from the intensified camera (Fig. $5 A$ ) or any of the pictures in false colour (Fig. 5B) can be used in the FluorCam software to select the areas of interest for further processing, such as plotting the time course of fluorescence yield. In the present case the three Micrasterias cells have been selected (see ellipses in the panels of Fig. $5 B$ ) to show kinetics of the fluorescence yield values for averages of all pixels in each of the cells (Fig. 5C). The course of $\Phi_{F}$ curves is noticeably different due to differences in cell age.

Fig. 6 presents, in a similar formal arrangement, the analysis of data recorded on coenobia of Scenedesmus quadricauda but does not include the black-and-white pictures corresponding to Fig. $5 A$. In contrast to Fig. 5 , the distribution of $\left.\Phi_{\mathrm{PS} 2}=\left(\mathrm{F}_{\mathrm{M}}-\mathrm{F}_{0}\right) / \mathrm{F}_{\mathrm{M}}\right)$ values is here displayed only for the pixels within the areas of the cells. These recordings show that the microscope is able to demonstrate identical and differing characters in distinct coenobia. The different shape of the curves corresponds to different stages of the cell cycle.

Another example of the differences due to cell development is the record of somatic cells as compared to sporangia in the filamentous brown alga Ectocarpus siliculosus (Fig. 7). The analysis refers to the cells illustrated in the black-and-white Fig. 3. The maps in Fig. $7 A$ and the curves in Fig. $7 B$ show that fluorescence parameters and kinetics differ noticeably between the cells in different life cycle stages of the alga. By keeping the same filament in the measuring chamber providing a controlled environment over several days, it is even possible to follow the course of development in a sporangium. From another angle of view the analysis of Fig. 7 demonstrates that individual chloroplasts selected in the algae cell yield reasonable kinetic curves (small insert in Fig. 7B).

The next example shows Elodea leaves with epiphytic diatoms (Fig. 8). The two organisms show a clear-cut difference both in the shape of the transient curves and in the fluorescence intensity. The latter difference, which is outstanding mainly in the images on Fig. $8 A$, is in the graph suppressed by the normalisation of both curves according to their $F_{0}$ values. This example shows further, that using our device it is easily possible to perform, on the microscopic level, the comparative in situ analysis (e.g., for ecological studies) of photosynthetic performance in various members of a plant community under strictly comparable conditions.

With chloroplasts in some plants we met a difficulty, which has to be kept in mind in planning experiments. At the same time it may enrich the investigation of chloroplast movements in response to actinic irradiance. 
When we took imaging records of cells in the leaves of Elodea canadensis (Fig. 9) with the protocol including $50 \mathrm{~s}$ interval of actinic irradiation, the chloroplasts in the cells moved from their dark adapted position to the strong irradiance position during this interval. The latter becomes evident by comparing the position of the chloroplasts in the image taken before actinic irradiance is switched on $\left(\mathrm{F}_{0}\right.$ record, Fig. $9 A$, bottom right panel) and immediately after it is switched off $\left(\mathrm{F}_{0}\right.$ ' in Fig. $9 A$, top left). An opposite movement takes place during the following dark period. The image $\mathrm{F}_{0}$ " (Fig. 9A, top right) recorded at the end of this period shows that some of the chloroplasts returned to the middle of the cell, although certainly not to exactly the same position as before. We have taken the value of the ratio $F_{0}{ }^{\prime}{ }^{\prime} / F_{0}$ ' as measure of the movement. This experience shows that the values recorded and displayed as the transient of $F_{0}$ upon lightdark transition $\left(\mathrm{F}_{0}\right.$ ' to $\left.\mathrm{F}_{0}{ }^{\prime \prime}\right)$ result, at any given time, from both the changes in $\Phi_{F}$ and the chloroplast movement away from or into the area selected for evaluation. Specifically, the very often discussed value of $F_{0}$ ' reflects, in this case, the effect of both factors mentioned.

At low magnification we performed test measurements with young, healthy leaves of Hibiscus. Fig. 10 shows one such record which reveals differences in photosynthetic performance (Fig. 10A) and in kinetics of

\section{References}

Bilger, W., Schreiber, U.: Chlorophyll luminescence as an indicator of stress-induced damage to the photosynthetic apparatus. Effects of heat-stress in isolated chloroplasts. Photosynth. Res. 25: 161-171, 1990.

Bowyer, W.J., Ning, L., Daley, L.S., Strobel, G.A., Edwards, G.E., Callis, J.B.: In vivo fluorescence imaging for detection of damage to leaves by fungal phytotoxins. - Spectroscopy 13(11): 36-44, 1998.

Buschmann, C., Lichtenthaler, H.K.: Principles and characteristics of multi-colour fluorescence imaging of plants. - J. Plant Physiol. 152: 297-314, 1998.

Cardon, Z.G., Mott, K.A., Berry, J.A.: Dynamics of patchy stomatal movements, and their contribution to steady-state and oscillating stomatal conductance calculated using gasexchange techniques. - Plant Cell Environ. 17: 995-1007, 1994.

Daley, P.F.: Chlorophyll fluorescence analysis and imaging in plant stress and disease. - Can. J. Plant Pathol. 17: 167-173, 1995.

Daley, P.F., Raschke, K., Ball, J.T., Berry, J.A.: Topography of photosynthetic activity of leaves obtained from video images of chlorophyll fluorescence. - Plant Physiol. 90: 1233-1238, 1989.

Dau, H.: Molecular mechanisms and quantitative models of variable photosystem II fluorescence. - Photochem. Photobiol. 60: 1-23, 1994.

Fenton, J.M., Crofts, A.R.: Computer aided fluorescence imaging of photosynthetic systems: Application of video imaging to the study of fluorescence induction in green plants fluorescence transients (Fig. 10B) between more active parts of the mesophyll and less active ones in the sectors around the vein and at the edge of the leaf. The biological relevance of these differences is an interesting subject for further studies. We present this demonstration here to show that if for some investigation the macroscopic FluorCam device does not yield the results with a satisfactory spatial resolution, the microscopic variant can be applied with advantage on this borderline between macro- and micro-fluorescence imaging.

Conclusions: Our system provides a so far not reported capacity to record, on a microscopic scale, the complete two-dimensional kinetics of in vivo $\mathrm{Chl}$ fluorescence using pulsed measuring radiation. Records of fluorescence transients caused by various regimes of actinic irradiation can be obtained together with an exact on line record of $F_{0}$ and $F_{0}$, and it is also possible to perform the quenching analysis with saturation flashes. All this is possible in a wide range of size scales, from small parts of leaves down to single cells and chloroplasts. At present, limitations are mainly caused by high noise and bad spatial resolution of the image intensifier as well as by the limited output of blue LEDs used for the exciting measuring pulses. and photosynthetic bacteria. - Photosynth. Res. 26: 59-66, 1990.

Govindjee: Sixty-three years since Kautsky: Chlorophyll $a$ fluorescence. - Aust. J. Plant Physiol. 22: 131-160, 1995.

Jensen, M., Siebke, K.: Fluorescence imaging of lichens in the macro scale. - Symbiosis 23: 183-195, 1997.

van Kooten, O., Snel, J.F.H.: The use of chlorophyll fluorescence nomenclature in plant stress physiology. Photosynth. Res. 25: 147-150, 1990.

Krause, G.H., Weis, E.: Chlorophyll fluorescence and photosynthesis: The basics. - Annu. Rev. Plant Physiol. Plant mol. Biol. 42: 313-349, 1991.

Lichtenthaler, H.K., Lang, M., Sowinska, M., Heisel, F., Miehé, J.A.: Detection of vegetetion stress via a new high resolution fluorescence imaging system. - J. Plant Physiol. 148: 599$612,1996$.

Lootens, P., Vandecasteele, P.: A cheap chlorophyll a fluorescence imaging system. - Photosynthetica 38: 53-56, 2000.

Meyer, S., Genty, B.: Mapping intercellular $\mathrm{CO}_{2}$ mole fraction (Ci) in Rosa rubiginosa leaves fed with abscisic acid by using chlorophyll fluorescence imaging. Significance of Ci estimated from leaf gas exchange. - Plant Physiol. 116: 947 957, 1998.

Nedbal, L., Soukupová, J., Kaftan, D., Whitmarsh, J., Trtílek, M.: Kinetic imaging of chlorophyll fluorescence using modulated light. - Photosynth. Res., in press, 2001.

Omasa, K., Shimazaki, K.-I., Aiga, I., Larcher, W., Onoe, M.: Image analysis of chlorophyll fluorescence transients for diagnosing the photosynthetic system of attached leaves. - 
Plant Physiol. 84: 748-752, 1987.

Osmond, B., Schwartz, O., Gunning, B.: Photoinhibitory printing on leaves, visualised by chlorophyll fluorescence imaging and confocal microscopy, is due to diminished fluorescence from grana. - Aust. J. Plant Physiol. 26: $717-$ $724,1999$.

Oxborough, K., Baker, N.R.: Resolving chlorophyll $a$ fluorescence images of photosynthetic efficiency into photochemical and non-photochemical components calculation of $q P$ and $F v^{\prime} / F_{M}$ ' without measuring $F_{0}$ '. Photosynth. Res. 54: 135-142, 1997a.

Oxborough, K., Baker, N.R.: An instrument capable of imaging chlorophyll $a$ fluorescence from intact leaves at very low irradiance and at cellular and subcellular levels of organization. - Plant Cell Environ. 20: 1473-1483, $1997 \mathrm{~b}$.

Rolfe, S.A., Scholes, J.D.: Quantitative imaging of chlorophyll fluorescence. - New Phytol. 131: 69-79, 1995.

Schreiber, U.: Chlorophyll fluorescence: new applications. - In:
Garab, G. (ed.) Photosynthesis: Mechanisms and Effects. Vol. V. Pp. 4253-4258. Kluwer Academic Publ., Dordrecht Boston - London 1998.

Schreiber, U., Hormann, H., Neubauer, C., Klughammer, C.: Assessment of photosystem II photochemical quantum yield by chlorophyll fluorescence quenching analysis. - Aust. J. Plant Physiol. 22: 209-220, 1995.

Schreiber, U., Neubauer, C., Schliwa, U.: PAM fluorometer based on medium-frequency pulsed Xe-flash measuring light: A highly sensitive new tool in basic and applied photosynthetic research. - Photosynth. Res. 36: 65-72, 1993.

Schreiber, U., Schliwa, U., Bilger, W.: Continuous recording of photochemical and non-photochemical chlorophyll fluorescence quenching with a new type of modulation fluorometer. - Photosynth. Res. 10: 51-62, 1986.

Siebke, K., Weis, E.: Imaging of chlorophyll- $a$-fluorescence in leaves: Topography of photosynthetic oscillations in leaves of Glechoma hederacea. - Photosynth. Res. 45: 225-237, 1995. 